\title{
Direct Simulation of Low-Re Flow around a Square Cylinder by Numerical Manifold Method for Navier-Stokes Equations
}

\author{
Zhengrong Zhang and Xiangwei Zhang \\ School of Materials and Energy, Guangdong University of Technology, Guangzhou 510643, China \\ Correspondence should be addressed to Zhengrong Zhang, zzr@gdut.edu.cn
}

Received 15 January 2012; Accepted 3 August 2012

Academic Editor: Fu-Yun Zhao

Copyright (c) 2012 Z. Zhang and X. Zhang. This is an open access article distributed under the Creative Commons Attribution License, which permits unrestricted use, distribution, and reproduction in any medium, provided the original work is properly cited.

Numerical manifold method was applied to directly solve Navier-Stokes (N-S) equations for incompressible viscous flow in this paper, and numerical manifold schemes for N-S equations coupled velocity and pressure were derived based on Galerkin weighted residuals method as well. Mixed cover with linear polynomial function for velocity and constant function for pressure was employed in finite element cover system. As an application, mixed cover 4-node rectangular manifold element has been used to simulate the incompressible viscous flow around a square cylinder in a channel. Numerical tests illustrate that NMM is an effective and high-order accurate numerical method for incompressible viscous flow N-S equations.

\section{Introduction}

In computational fluid dynamics (CFD), Navier-Stokes (N-S) equations for incompressible viscous flow can be solved by several numerical methods generally, such as finite difference method (FDM), finite element method (FEM), and finite volume method (FVM) [1]. The applications of FDM are affected for unsuitability to complicated structure flow field and inconservation of field variables [2]. The FEM schemes for N-S equations are very complicated, and the computational efficiency of nonstandard FEMs is low [3]. FVM is an effective numerical method for solutions of fluid flow, but exact treatments of the moving boundary are difficult and complicated [4]. Furthermore, fractional step algorithms are used to solve velocity and pressure variables unintegratedly in all these numerical methods, and the continuity equation and momentums equations are satisfied, respectively [5]. All the defects of these numerical methods will affect the computational efficiency and accuracy. 
Numerical manifold method (NMM) also known as manifold method or finite cover method (FCM) is a generalized numerical method proposed by Shi in the early 1990s [6, 7]. The method performs numerical computation with finite element cover system, which is composed of two independent cover grids: mathematical cover grid and physical cover grid. Mathematical covers define the accuracy of approximate solution, and physical covers determine the solution domain. High accurate numerical manifold schemes for mathematical physics equations can be constructed by adopting different cover functions and coverweighted functions for physical variables in finite element cover system [8]. The method has been successfully applied in some complicated engineering problems, such as numerical simulation of crack initiation and propagation, damage evolvement of fractured rock masses, coupling vibration, and potential flow problem, and the advantages have been demonstrated in these fields [9-12]. As to fluid flow problems, direct-numerical-solution coupled velocity and pressure variables can be implemented by adopting manifold schemes with mixed cover for N-S equations. The authors have applied numerical manifold method into analyses of unsteady incompressible viscous flow, and the results have illustrated the validity of NMM [13].

Flow around a square cylinder is a typical model to validate the performance of numerical methods for solution of incompressible viscous N-S equations. The flow structure has been investigated experimentally and numerically. Experimental investigations have shown that the flow characteristic is different at different Reynolds numbers [14]. FVM, FDM, and others numerical methods have been applied to simulate the flow around a square cylinder, the detail results have been obtained and met the experimental results [15-17]. The experimental and numerical results have been employed to validate the numerical methods in CFD as benchmarks.

In this paper, numerical manifold schemes of direct solutions coupled velocity and pressure for N-S equations were constructed and applied to analyze incompressible viscous flow around a square cylinder in a parallel channel. The validations of numerical schemes to steady and unsteady flow were completed.

\section{Numerical Manifold Schemes for N-S Equations of Incompressible Viscous Flow}

\subsection{Galerkin Integration Expressions of N-S Equations}

For numerical solution of incompressible viscous flow, the integration expressions for the continuity equation and N-S equations can be obtained by Galerkin weighted residual method, and the weak solution form can be stated as

$$
\begin{gathered}
\int_{\Omega} v_{j} \frac{\partial}{\partial x_{j}}(\delta p) \mathrm{d} \Omega=\int_{\Gamma_{1}} \bar{v}_{n} \delta p \mathrm{~d} \Gamma \\
\int_{\Omega}\left[\rho\left(\frac{\partial v_{i}}{\partial t}+v_{j} \frac{\partial v_{i}}{\partial x_{j}}\right) \delta v_{i}+\left[-p \delta_{i j}+\mu\left(\frac{\partial v_{i}}{\partial x_{j}}+\frac{\partial v_{j}}{\partial x_{i}}\right)\right] \frac{\partial}{\partial x_{j}}\left(\delta v_{i}\right)\right] \mathrm{d} \Omega \\
=\int_{\Gamma_{2}} \bar{p}_{i} \delta v_{i} \mathrm{~d} \Gamma+\int_{\Omega} \rho f_{i} \delta v_{i} \mathrm{~d} \Omega
\end{gathered}
$$


where $t$ denotes the time variable; $\rho$ is the fluid density; $\mu$ is the dynamic viscosity; $v_{j}$ is the velocity in $x_{j}$ direction; $p$ is the pressure; $f_{i}$ is the body force per unit volume in $x_{i}$ direction; $\delta p$ is referred to the weight function for continuity equation and $\delta v_{i}$ for momentum equation; $\Omega$ is the domain of solution; $\bar{v}_{n}$ is the known normal velocity on boundary $\Gamma_{1} ; \bar{p}_{i}$ is the known pressure components in $x_{i}$ direction on boundary $\Gamma_{2}$.

\subsection{Numerical Manifold Schemes for N-S Equations}

\subsubsection{Element Velocity and Pressure Cover Functions}

When NMM is applied into solution of N-S equations, suggested that there are $M$ manifold elements in finite element cover system and each element $(e)$ has $n$ physical covers, and the cover weight functions are $N_{j}(x, y)(j=1,2, \ldots, n)$, which meet the requirement of partition of unity $\sum_{j=1}^{n} N_{j}(x, y)=1(x, y \in e)$, then the element global velocity and pressure cover functions $v_{j}(x, y)$ and $p(x, y)$ can be given by weighted summation of the cover functions of every physical cover as the follows:

$$
\begin{gathered}
v_{i}(x, y)=N_{j}(x, y) v_{i j}(x, y), \\
p(x, y)=N_{j}(x, y) p_{j}(x, y),
\end{gathered}
$$

where $i=1,2$ for two-dimensional (2D) flow problem; $v_{i j}(x, y)$ are the velocity component cover functions for physical cover $U_{j}$ of element $(e)$ and $p_{j}(x, y)$ is the pressure cover function. If polynomial functions are taken as the cover functions of every physical cover, the cover functions can be stated in the form as

$$
\begin{gathered}
v_{i j}(x, y)=f_{k}^{v} \cdot D_{i j k^{\prime}}^{v} \\
p_{j}(x, y)=f_{l}^{p} \cdot D_{j l^{\prime}}^{p}
\end{gathered}
$$

where $f_{k}^{v}, f_{l}^{p}$ denote the basic series for velocity and pressure cover functions of physical cover $U_{j} ; D_{i j k}^{v}, D_{j l}^{p}$ are the cover DOF variables, and the DOF numbers are $m_{v}\left(k=1,2, \ldots, m_{v}\right)$ and $m_{p}\left(l=1,2, \ldots, m_{p}\right)$.

By substituting (2.3) into (2.2), the global cover functions for velocity and pressure in element $(e)$ can be written as

$$
\begin{gathered}
v_{i}(x, y)=N_{j}(x, y) v_{i j}(x, y)=N_{j}(x, y) f_{k}^{v} D_{i j k}^{v}=T_{j k}^{v} D_{i j k^{\prime}}^{v} \\
p(x, y)=N_{j}(x, y) p_{j}(x, y)=N_{j}(x, y) f_{l}^{p} D_{j l}^{p}=T_{j l}^{p} D_{j l^{\prime}}^{p}
\end{gathered}
$$

where $T_{j k}^{v}$ is the element velocity cover basic function; $T_{j l}^{p}$ is the element pressure cover basic function. The element cover basic functions consist of the basic series of cover functions and cover weight functions, and the element DOF variables are composed of all the cover DOF variables. 
In Galerkin integration equations (2.1a) and (2.1b), the weight functions for continuity equation and N-S equations can adopt the element basic functions of velocity components and pressure accordingly, that is,

$$
\delta v_{i}=T_{j k}^{v} ; \quad \delta p=T_{j l}^{p}
$$

The partial derivatives of element cover functions can be obtained from (2.4a) and $(2.4 b)$ as the follows:

$$
v_{i, \alpha}=T_{j k, \alpha}^{v} D_{i j k}^{v} ; \quad p_{, \alpha}=T_{j l, \alpha}^{p} D_{j l}^{p} ; \quad v_{i, t}=T_{j k}^{v} D_{i j k, t}^{v}=T_{j k}^{v} \dot{D}_{i j k}^{v}
$$

where $\alpha=1,2$ for 2D problem; $T_{j k, \alpha^{\prime}}^{v} T_{j l, \alpha}^{p}$ are the partial derivatives of element velocity and pressure cover basic functions with respect to $x_{\alpha}$, respectively; $\dot{D}_{i j k}^{v}$ are the partial derivatives of element velocity DOF variables with respect to $t$.

\subsubsection{Element Manifold Schemes}

For each element $(e)$ in finite element cover system, element manifold equations can be derived by introducing (2.4a), (2.4b), (2.5), and (2.6) into (2.2), and the equations can be stated as

$$
\begin{gathered}
\int_{\Omega^{e}} T_{\beta I}^{v} D_{\alpha \beta I}^{v} T_{j l, \alpha}^{p} \mathrm{~d} \Omega=\int_{\Gamma_{1}^{e}} \bar{v}_{n} T_{j l}^{p} \mathrm{~d} \Gamma \\
\int_{\Omega^{e}}\left[\rho T_{\beta I}^{v} \dot{D}_{i \beta I}^{v} T_{j k}^{v}+\rho T_{\beta I}^{v} D_{\alpha \beta I}^{v} T_{\gamma J, \alpha}^{v} D_{i \gamma J}^{v} T_{j k}^{v}-T_{\beta K}^{p} D_{\beta K}^{p} \delta_{i \alpha} T_{j k, \alpha}^{v}+\mu\left(T_{\gamma J, \alpha}^{v} D_{i \gamma J}^{v}+T_{\gamma J, i}^{v} D_{\alpha \gamma J}^{v}\right) T_{j k, \alpha}^{v}\right] \mathrm{d} \Omega \\
=\int_{\Gamma_{2}^{e}} \bar{p}_{i} T_{j k}^{v} \mathrm{~d} \Gamma+\int_{\Omega^{e}} \rho f_{i} T_{j k}^{v} \mathrm{~d} \Omega,
\end{gathered}
$$

that is,

$$
\begin{gathered}
G_{j l \alpha \beta I}^{e} D_{\alpha \beta I}^{v}=H_{j l^{\prime}}^{e} \\
A_{j k \gamma J}^{e} \dot{D}_{i \gamma J}^{v}+B_{j k \alpha \beta Y I J}^{e} D_{\alpha \beta I}^{v} D_{i \gamma J}^{v}+C_{i j k \beta K}^{e} D_{\beta K}^{p}+E_{j k i \alpha \gamma J}^{e} D_{\alpha \gamma J}^{v}=F_{i j k}^{e}
\end{gathered}
$$

where $\beta, \gamma=1,2, \ldots, n ; I, J=1,2, \ldots, m_{v}, K=1,2, \ldots, m_{p}$; and the coefficients for element manifold equations are

$$
\begin{aligned}
& A_{j k \gamma J}^{e}=\int_{\Omega^{e}} \rho T_{\gamma J}^{v} T_{j k}^{v} \mathrm{~d} \Omega, \quad B_{j k \alpha \beta \gamma I J}^{e}=\int_{\Omega^{e}} \rho T_{\beta I}^{v} T_{\gamma J, \alpha}^{v} T_{j k}^{v} \mathrm{~d} \Omega, \\
& C_{i j k \beta K}^{e}=-\int_{\Omega^{e}} T_{\beta K}^{p} \delta_{i \alpha} T_{j k, \alpha}^{v} \mathrm{~d} \Omega, \quad E_{j k i \alpha \gamma J}^{e}=\int_{\Omega^{e}} \mu T_{j k, s}^{v}\left(\delta_{i \alpha} T_{\gamma J, S}^{v}+\delta_{s \alpha} T_{\gamma J, i}^{v}\right) \mathrm{d} \Omega, \\
& F_{i j k}^{e}=\int_{\Gamma_{2}^{e}} \bar{p}_{i} T_{j k}^{v} \mathrm{~d} \Gamma+\int_{\Omega^{e}} \rho f_{i} T_{j k}^{v} \mathrm{~d} \Omega, \quad G_{j l \alpha \beta I}^{e}=\int_{\Omega^{e}} T_{\beta I}^{v} T_{j l, \alpha}^{p} \mathrm{~d} \Omega, \quad H_{j l}^{e}=\int_{\Gamma_{1}^{e}} \bar{v}_{n} T_{j l}^{p} \mathrm{~d} \Gamma .
\end{aligned}
$$


Equations (2.8a) and (2.8b) are the nonlinear algebra equations with respect to coupled velocity and pressure DOF variables and first-order linear ordinary differential equations with respect to time.

\section{Some Key Techniques}

\subsection{Mixed Cover Functions for Velocity and Pressure}

In FEM, when velocity and pressure field are discretized in the same way, the discrete element can not be ensured to satisfy the Ladyzanskya Babuska Brezzi stability condition (LBB condition), and these kinds of element cannot be applied to directly solve N-S equations coupled velocity and pressure for the spatial oscillation of pressure field. Mixed elements can be built to meet the LBB condition by increasing velocity interpolation node [18], but the elements are inconvenient to apply in practice for the complicated calculation process and difficult element discretization.

In finite element cover system of NMM, the element cover functions are composed of the weight functions and the cover functions of physical covers. Generally, the weight functions will adopt the element shape functions as in FEM, which are usually defined by the element shape and nodes. The cover functions of every physical cover for different physical variables can employ different order functions according to the solving physical equations. When NMM is applied to solve N-S equations for incompressible viscous flow, the velocity and pressure field can be discretized in the same way and the weight functions will employ the same interpolation functions for element velocity and pressure variables. But, the cover functions of every element physical cover can apply different order functions for velocity and pressure variables, so it will form a manifold element with mixed cover functions for velocity and pressure variables, which can meet the requirements of different order approximate functions for velocity and pressure in Galerkin integration expressions (2.1a) and (2.1b) and satisfy the LBB condition for increasing the DOF numbers of velocity variables. The mixed cover manifold elements can be applied in direct solution of incompressible viscous N-Sequations-coupled velocity and pressure variables.

In theory, velocity components and pressure covers can employ very high-order functions, but it will cause a very complicated calculation process, so low-order polynomial functions are favorable. If pressure cover function of every physical cover is defined as constant function, velocity cover functions can adopt linear polynomial functions, and then the basic series in (2.3) can be given by the following localized series for 2D problem:

$$
\left[f_{k}^{v}\right]=\left[\begin{array}{llll}
1 & \eta_{1}\left(x-x_{c}\right) & \eta_{2}\left(y-y_{c}\right)
\end{array}\right] ; \quad\left[f_{l}^{p}\right]=[1],
$$

where $(x, y)$ is the coordinate variables; $\left(x_{c}, y_{c}\right)$ is the center coordinates of physical cover; $\eta_{1}, \eta_{2}$ are the coefficients of cover functions.

\subsection{Solution of Manifold Equations for Steady N-S Equations}

As to $2 \mathrm{D}$ steady N-S equations, element manifold equations (2.8a) and (2.8b) can be reformatted as

$$
B_{j k \alpha \beta Y I J}^{e} D_{\alpha \beta I}^{v} D_{1 \gamma J}^{v}+C_{1 j k \beta K}^{e} D_{\beta K}^{p}+E_{j k 1 \alpha \gamma J}^{e} D_{\alpha \gamma J}^{v}=F_{1 j k}^{e}
$$




$$
\begin{gathered}
B_{j k \alpha \beta \gamma I J}^{e} D_{\alpha \beta I}^{v} D_{2 \gamma J}^{v}+C_{2 j k \beta K}^{e} D_{\beta K}^{p}+E_{j k 2 \alpha \gamma J}^{e} D_{\alpha \gamma J}^{v}=F_{2 j k^{\prime}}^{e} \\
G_{j l \alpha \beta I}^{e} D_{\alpha \beta I}^{v}=H_{j l^{\prime}}^{e}
\end{gathered}
$$

where $j, k, l, \alpha, \beta, \gamma, I, J$ are similar to (2.8a) and (2.8b); $D_{1 \gamma J}, D_{2 \gamma J}$, and $D_{\beta K}$ are the unknown variables. The equations can be written in the matrix form as

$$
\left[\begin{array}{ccc}
B_{j k 1 \beta \gamma I J}^{e} D_{1 \beta I}^{v}+E_{j k 11 \gamma J}^{e} & B_{j k 2 \beta \gamma I J}^{e} D_{1 \beta I}^{v}+E_{j k 12 \gamma J}^{e} & C_{1 j k \beta K}^{e} \\
B_{j k 1 \beta \gamma I J}^{e} D_{2 \beta I}^{v}+E_{j k 21 \gamma J}^{e} & B_{j k 2 \beta \gamma I J}^{e} D_{2 \beta I}^{v}+E_{j k 22 \gamma J}^{e} & C_{2 j k \beta K}^{e} \\
G_{j l 1 \gamma J}^{e} & G_{j l 2 \gamma J}^{e} & 0
\end{array}\right]\left\{\begin{array}{c}
D_{1 \gamma J}^{v} \\
D_{2 \gamma J}^{v} \\
D_{\beta K}^{p}
\end{array}\right\}=\left\{\begin{array}{c}
F_{1 j k}^{e} \\
F_{2 j k}^{e} \\
H_{j l}^{e}
\end{array}\right\},
$$

that is,

$$
\left[K^{e}\right]\left\{D^{e}\right\}=\left\{F^{e}\right\}
$$

where $\left[K^{e}\right]$ is the element flow matrix with respect to unknown velocity DOF variables; $\left\{D^{e}\right\}$ is the element DOF variables; $\left\{F^{e}\right\}$ is the element generalized loads.

The manifold equations (3.3a) and (3.3b) are nonlinear algebra equations and can be solved by two methods of directly linearizing alternate iteration and Newton-Raphson iterative in general.

\subsection{Solution of Manifold Equations for Steady N-S Equations}

As to $2 \mathrm{D}$ unsteady N-S equations, the first-order linear ordinary differential equation (2.8b) with respect to time need be translated into linear algebra equations by time difference replacing the derivative. At any discrete time step $t_{i}$, the nonlinear manifold equations can be solved by implicit iterative methods or explicit methods. If implicit iterative methods are used, the derivative of velocity DOF variables for every physical cover with respect to time in $(2.8 \mathrm{~b})$ will be discretized by time difference as

$$
\dot{D}_{i j k}^{v}=\frac{\left(D_{i j k}^{v(i+1)}-D_{i j k}^{v(i)}\right)}{\Delta t},
$$

where $\Delta t$ is time step; $D_{i j k}^{v(i)}$ and $D_{i j k}^{v(i+1)}$ are the velocity DOF variables at time step $t_{i}$ and $t_{i+1}$, respectively. Then as to every manifold element $(e)$, the element manifold equations $(2.8 \mathrm{~b})$ can be reformulated as the follows:

$$
\begin{aligned}
& A_{j k \gamma J}^{e} D_{i \gamma J}^{v(i+1)}+\Delta t B_{j k \alpha \beta \gamma I J}^{e} D_{\alpha \beta I}^{v(i+1)} D_{i \gamma J}^{v(i+1)}+\Delta t C_{i j k \beta K}^{e} D_{\beta K}^{p(i+1)}+\Delta t E_{j k i \alpha \gamma J}^{e} D_{\alpha \gamma J}^{v(i+1)} \\
& \quad=\Delta t F_{i j k}^{e}+A_{j k \beta I}^{e} D_{i j k}^{v(i)} .
\end{aligned}
$$


Equations (3.5) uniting (2.8a) can be stated in matrix form as

$$
\begin{gathered}
{\left[\begin{array}{ccc}
A_{j k \gamma J}^{e}+\Delta t\left(B_{j k 1 \beta \gamma I J}^{e} D_{1 \beta I}^{v(i+1)}+E_{j k 11 \gamma J}^{e}\right) & \Delta t\left(B_{j k 2 \beta \gamma I J}^{e} D_{1 \beta I}^{v(i+1)}+E_{j k 12 \gamma J}^{e}\right) & \Delta t C_{1 j k \beta K}^{e} \\
\Delta t\left(B_{j k 1 \beta \gamma I J}^{e} D_{2 \beta I}^{v(i+1)}+E_{j k 21 \gamma J}^{e}\right) & A_{j k \gamma J}^{e}+\Delta t\left(B_{j k 2 \beta \gamma I J}^{e} D_{2 \beta I}^{v(i+1)}+E_{j k 22 \gamma J}^{e}\right) & \Delta t C_{2 j k \beta K}^{e} \\
G_{j l 1 \gamma J}^{e} & G_{j l 2 \gamma J}^{e} & 0
\end{array}\right]} \\
\cdot\left\{\begin{array}{l}
D_{1 \gamma J}^{v(i+1)} \\
D_{2 \gamma J}^{v(i+1)} \\
D_{\beta K}^{p(i+1)}
\end{array}\right\}=\left\{\begin{array}{c}
\Delta t F_{1 j k}^{e}+A_{j k \gamma J}^{e} D_{1 \gamma J}^{v(i)} \\
\Delta t F_{2 j k}^{e}+A_{j k \gamma J}^{e} D_{2 \gamma J}^{v(i)} \\
H_{j l}^{e}
\end{array}\right\},
\end{gathered}
$$

that is,

$$
\left[k^{e}\right]\left\{D^{e(i+1)}\right\}=\left\{F^{e}\right\}
$$

where $\left\{D_{\beta K}^{p(i+1)}\right\}$ is the pressure cover DOF variables at time step $t_{i+1} ;\left\{D^{e(i+1)}\right\}$ is the element unknown cover DOF variables at time step $t_{i+1} ;\left[k^{e}\right]$ is the element flow matrix with respect to the unknown velocity DOF variables at time step $t_{i+1} ;\left\{F^{e}\right\}$ is the element generalized loads with respect to the boundary conditions and the known velocity DOF variables at time step $t_{i}$.

The manifold equations (3.7) for unsteady flow can be solved by iterative methods. In this paper, Newton-Raphson iterative method is also used here for solution of unsteady flow manifold equations.

\section{Numerical Example of Flow around a Square Cylinder in a Channel}

To illustrate the validity of the present numerical manifold method, low-Re incompressible viscous flow around a square cylinder in a channel and flow past a step are investigated in details through direct solution of N-S equations in dimensionless form in this paper as two numerical examples. In numerical manifold analysis, standard rectangular finite element cover system with 4-node manifold element as show in Figure 1 is used, the mixed cover functions of physical covers with the basic series equations (3.1) are adopted, and the shape functions of rectangular element are employed as the cover weight functions in (2.2), that is,

$$
\begin{array}{ll}
N_{1}(x, y)=\frac{1}{4}\left(1-\frac{x-x_{0}}{a}\right)\left(1-\frac{y-y_{0}}{b}\right), & N_{2}(x, y)=\frac{1}{4}\left(1+\frac{x-x_{0}}{a}\right)\left(1-\frac{y-y_{0}}{b}\right), \\
N_{3}(x, y)=\frac{1}{4}\left(1+\frac{x-x_{0}}{a}\right)\left(1+\frac{y-y_{0}}{b}\right), & N_{4}(x, y)=\frac{1}{4}\left(1-\frac{x-x_{0}}{a}\right)\left(1+\frac{y-y_{0}}{b}\right),
\end{array}
$$

where $\left(x_{0}, y_{0}\right)$ are the coordinates of element (e) center point; $2 a \times 2 b$ are the side lengths. 


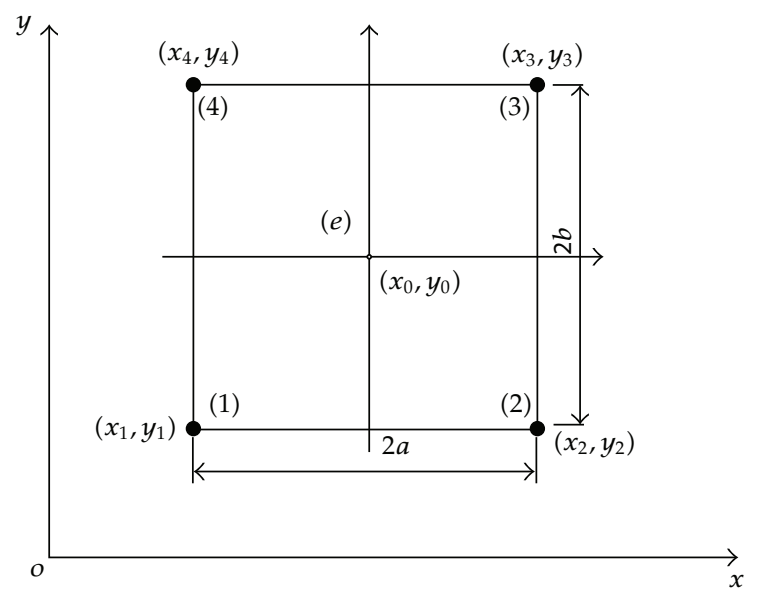

Figure 1: 4-node rectangular manifold element.

\subsection{Analysis for Steady Flow past a Step}

In numerical analysis of flow past a step, finite element cover system and boundary conditions are shown in Figure 2(a), the characteristic velocity of the flow field inlet is $u=1$ and $v=0$, the fluid density is $\rho=1$, and three dynamic viscosities of fluid are $\mu=0.01,0.005$, 0.0025 , and so Re numbers are known as $\operatorname{Re}=100,200,400$, respectively. All the flow field is meshed into 196 elements and 229 nodes by $0.1 \times 0.1$ rectangular grids.

The streamline and pressure distribution in flow field past a step at $\operatorname{Re}=200$ are shown in Figure 2(b). The streamline patterns obtained from direct numerical solutions have revealed the vortex structure at the step of flow field, and high accurate pressure distributions have been obtained as well. The numerical solutions from FVM are shown in Figures 2(c) and $2(\mathrm{~d})$ comparatively, which obtained from $0.1 \times 0.1$ and $0.05 \times 0.05$ rectangular grids, respectively. The NMM results from $0.1 \times 0.1$ grids are nearly coincident with the FVM results from $0.05 \times 0.05$ grids. The comparative analyses illustrate that numerical manifold schemes with mixed cover are stable numerical schemes for incompressible viscous flow, can implement direct numerical solution of N-S equations coupled velocity and pressure variables and can improve the solution accuracy at the same grids compared with FVM.

\subsection{Analysis for Steady Flow around a Square Cylinder in a Channel}

The flow field configuration and boundary conditions of flow around a square cylinder in a channel are shown in Figure 3. The length of the channel is $L=4$, the width is $W=1$. The size of square cylinder is $d \times d=0.25 \times 0.25$, its horizontal axis coincides with the channel's centerline, and its vertical axis is perpendicular to the channel's centreline. The velocity condition of the inlet $A D$ is $u / u_{\mathrm{av}}=1.5\left[1-\left(1-y / y_{m}\right)^{2}\right]\left(u_{\mathrm{av}}=1\right.$ is the average flow velocity in the channel, $y_{m}=0.5$ is the channel centerline) and $v=0$, the pressure condition of outlet $B C$ is $p=0$, and the nonslip boundary conditions are set on other fixed walls, that is, $u=v=0$.

The flow field is meshed by three mixed grids, one is that $32 \times 16$ uniform element grid is used for the domain from $x=0$ to $x=2$ and $20 \times 16$ element grid for the domain from $x=2$ 


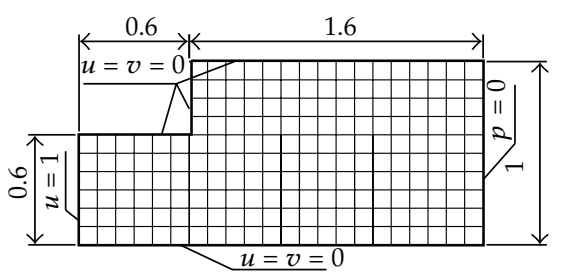

(a) Flow field configuration and boundary conditions
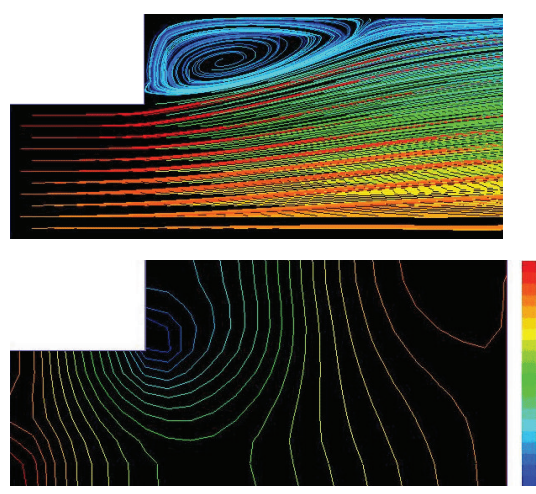

(c) FVM solutions $(0.1 \times 0.1$ grids $)$
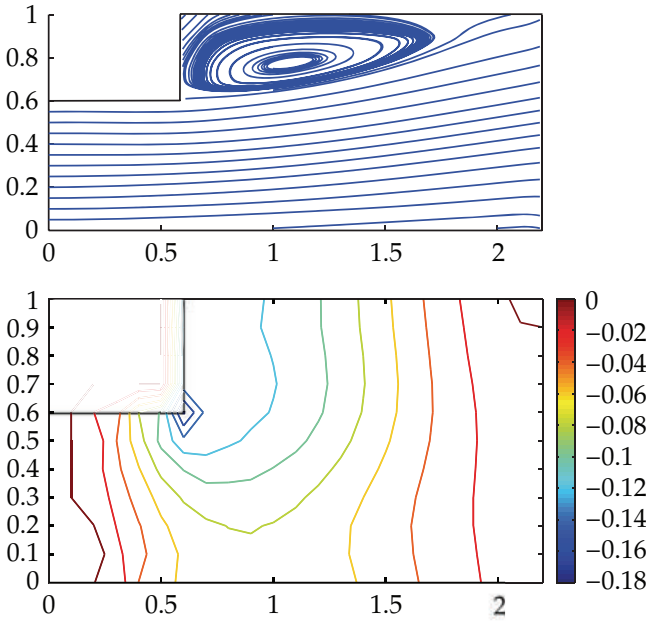

(b) NNM solutions $(0.1 \times 0.1$ grids $)$
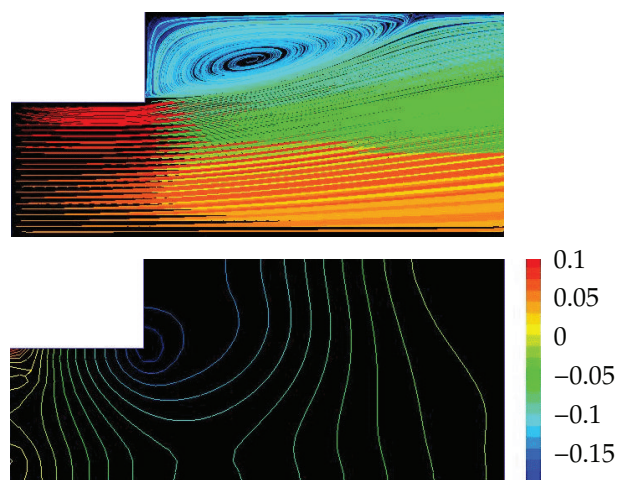

(d) FVM solutions $(0.05 \times 0.05$ grids $)$

Figure 2: The streamline patterns and pressure distributions for flow field past a step.

to $x=4$, the entire flow field is meshed by $52 \times 16=832$ elements and $53 \times 17=901$ nodes; the second one is that $64 \times 32$ uniform element grid is used for $x=0 \sim 2$ and $40 \times 32$ element grid for $x=2 \sim 4$, the flow field is meshed by $104 \times 32=3328$ elements and $105 \times 33=3465$ nodes; the third one is that $96 \times 48$ uniform element grid is used for $x=0 \sim 2$ and $64 \times 48$ element grid for $x=2 \sim 4$, the flow field is meshed by $160 \times 48=7680$ elements and $161 \times 49=7889$ nodes.

The numerical analyses of steady flow at different Re numbers $(\operatorname{Re}=25,50,75,100$, 150) are performed by the NMM solutions of steady N-S equations. The streamline patterns and variables distributions of flow field at $\mathrm{Re}=100$ from three different element grids are compared. The results from the first element grid are different to these from two other grids, but the results from the second grid are closely similar to these from the third one, and the average difference of velocity and pressure on the channel centreline is less than $5 \%$, so the second element grid is enough for flow at $\operatorname{Re}<100$, and the third element grid or finer element grid is more reasonable for flow at $\operatorname{Re}=100$ and 150 . 


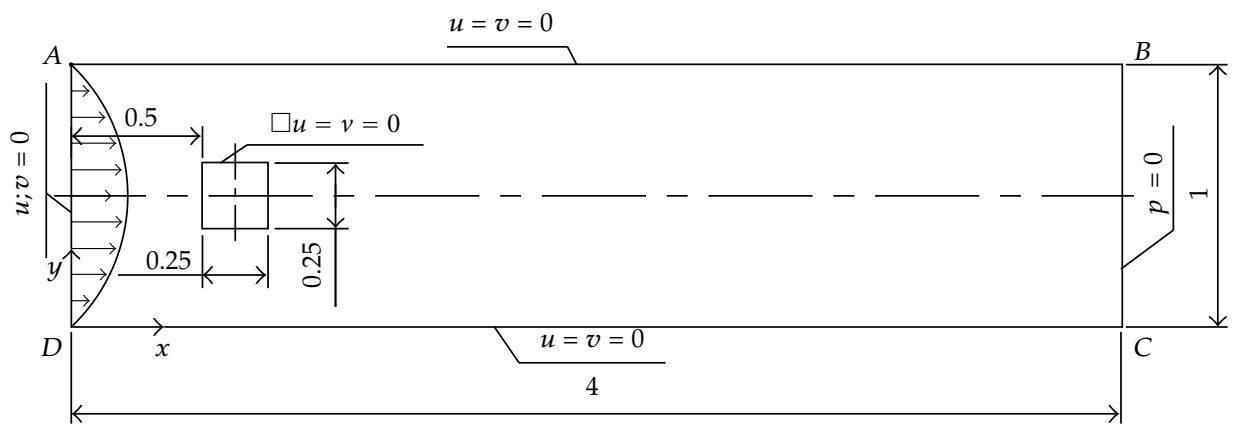

Figure 3: Flow field configuration and boundary conditions of flow around a square cylinder.
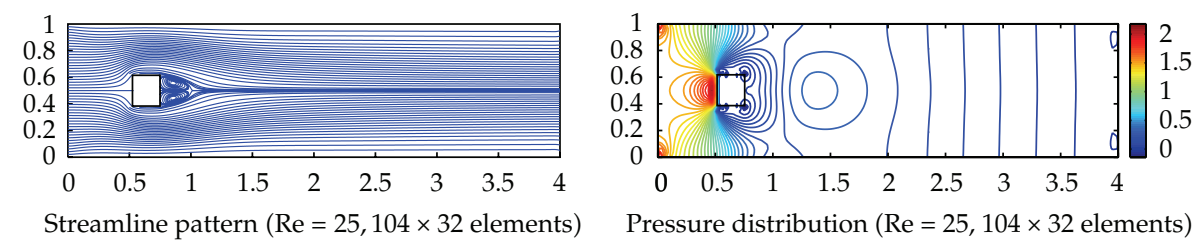

Streamline pattern $(\operatorname{Re}=25,104 \times 32$ elements $)$
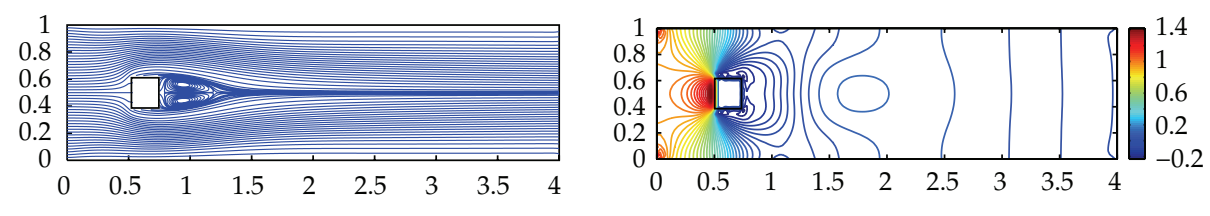

Streamline pattern $(\operatorname{Re}=50,104 \times 32$ elements $)$

Pressure distribution $(\mathrm{Re}=50,104 \times 32$ elements $)$
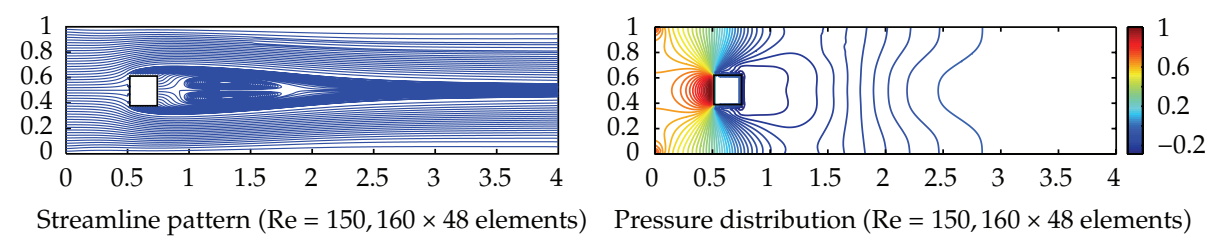

Figure 4: Streamline pattern and pressure distribution for flow field at different Re numbers.

The streamline patterns and pressure distributions at different Re numbers are shown in Figure 4. At $\operatorname{Re}=25$ and 50, there are two stable symmetrical vortexes behind the square cylinder, and the flow patterns are similar to the numerical solutions of Mukhopadhyay et al. [15]. As Re number increases to $\operatorname{Re} \approx[50,200]$, Cherry's results show that two vortexes become unsymmetrical and Karman vortex street is formed behind the square cylinder gradually [14], but the stable convergent results are obtained from NMM solutions of steady N-S equations at $\operatorname{Re}=75,100$, and 150, and there are also two stable symmetrical vortexes behind the square cylinder. The distances from back flow point of the vortexes to square cylinder at $\operatorname{Re}=25,50,75,100,150$ are 1.0, 1.2, 1.4, 1.6, 1.8, respectively, which are approximately linear with Re numbers. These results illustrate that numerical manifold schemes for steady N-S equations have the strong-stability-preserving property.

In NMM analysis unlike FVM and FEM analysis, the pressure distributions of flow field are obtained from direct numerical solutions of N-S equations and continuity equation, 


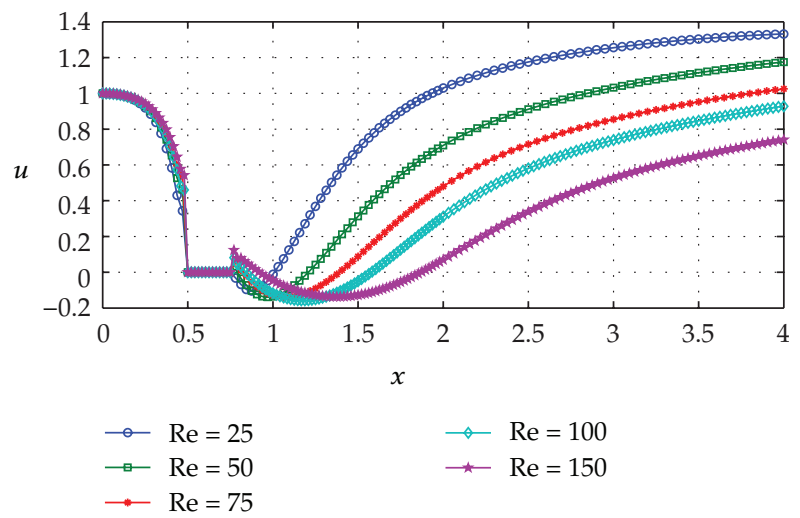

Figure 5: Comparison of velocity $u$ along horizontal lines through geometric center of flow field.

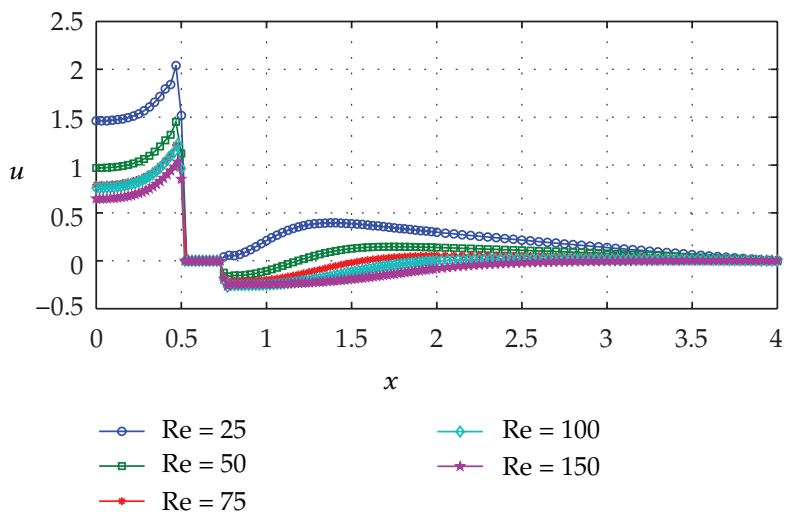

Figure 6: Comparison of pressure along horizontal lines through geometric center of flow field.

so it can improve the solution accuracy of pressure field. The pressure distributions of flow field show that high-pressure area is formed in frontage of the square cylinder for flow blockage, low pressure areas are formed near behind two front corner points for flow separating, and large pressure gradient is produced near the corner points. The pressure distributions are symmetrical, and the pressure of flow field will decrease as Re number increasing.

The velocity $u$ distributions along horizontal lines through geometric center of flow field are shown in Figure 5. The velocity $u$ drops rapidly from inlet to the square cylinder for flow blockage. Negative velocity appears behind the cylinder for back flow, and the velocity will rise gradually with away from the cylinder, and the velocity restoring becomes slow as Re number increases. The pressure distributions along horizontal lines through geometric center of flow field are shown in Figure 6. The pressure rises gradually from inlet to the square cylinder. Positive pressure appears behind the cylinder at low Re number, negative pressure appears at comparatively high Re number, and the pressure will drops or rises to zero at outlet of flow field. 


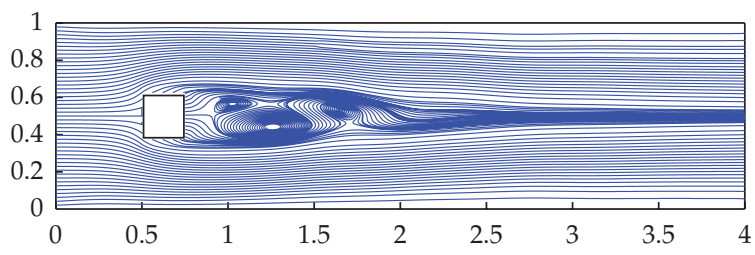

(a) $\mathrm{T} / 4$

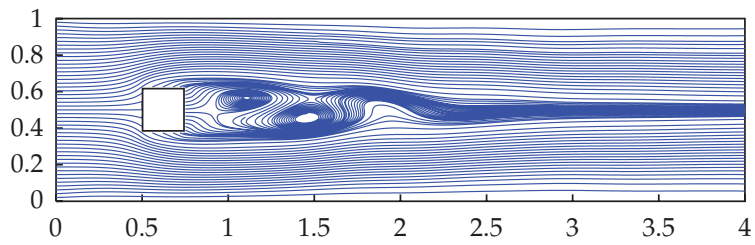

(b) $\mathrm{T} / 2$

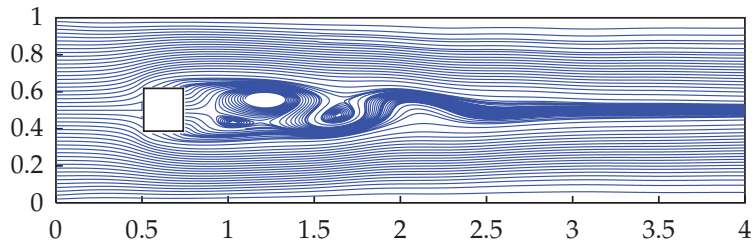

(c) $3 \mathrm{~T} / 4$

Figure 7: The streamline patterns for flow field from $48 \times 160$ element grid and $\Delta t=0.01 \mathrm{~s}$ time step at $\operatorname{Re}=$ 150.

\subsection{Analysis for Unsteady Flow around a Square Cylinder in a Channel}

The flow field configuration and boundary conditions of unsteady flow around a square cylinder in a channel are the same as these of steady flow, and the flow field is meshed by the third mixed grids. The unsteady flows at different Re numbers ( $\operatorname{Re}=50,75,100,150)$ are also analyzed through NMM solutions of N-S equations in this paper, where numerical solutions for steady flow at $\operatorname{Re}=50$ are adopted as the initial conditions, the total computational time is $10 \mathrm{~s}$, and the time step is $0.01 \mathrm{~s}$.

When $\operatorname{Re} \leq 50$, numerical solutions from unsteady flow analyses are closely similar to the results from steady flow analyses, and there are two steady symmetrical vortexes behind the square cylinder. As Re number increasing, two vortexes become unsymmetrical, and Karman Vortex Street is formed behind the square cylinder gradually. The streamline patterns for flow field at $\operatorname{Re}=150$ are shown in Figure 7, and the periodical change process of appearing, developing, moving, separating of the vortexes are clearly displayed in the streamline patterns. The Strouhal number is 0.2438 , the difference is $2.2 \%$ and $6.5 \%$ to $[15,16]$. The flow patterns are similar to Mukhopadhyay's and Yutaka's results, which is obtained from very fine element grid $[15,17]$.

The pressure contours for flow field at $\operatorname{Re}=150$ are show in Figure 8. The pressure distributions beside and in frontage of the square cylinder are almost identical to the results from steady flow analyses and are different behind the cylinder, which will change with the moving of vortexes. 


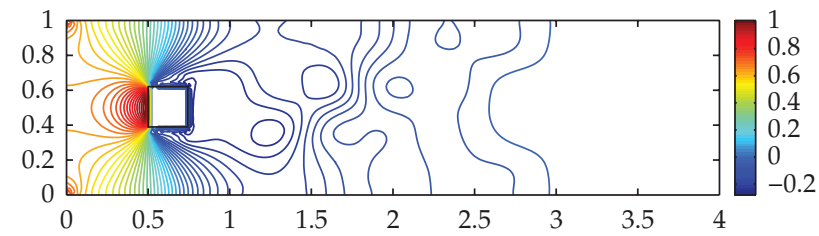

(a) $\mathrm{T} / 4$

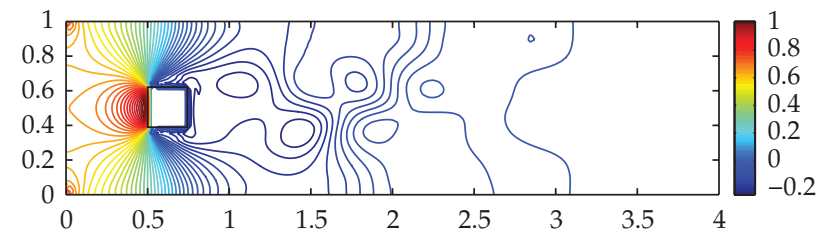

(b) $\mathrm{T} / 2$

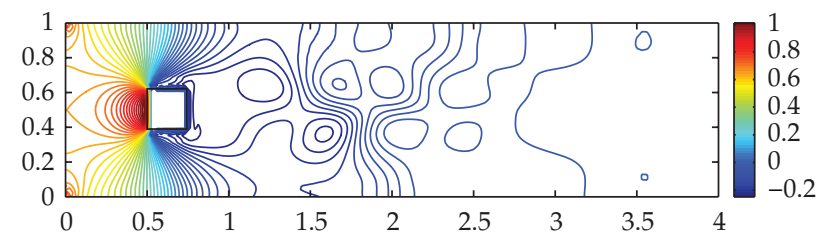

(c) $3 \mathrm{~T} / 4$

Figure 8: The pressure contours for flow field from $48 \times 160$ element grid and $\Delta t=0.01 \mathrm{~s}$ time step at $\operatorname{Re}=150$.

\section{Summary}

Numerical manifold method for direct coupled solution of incompressible viscous flow N-S equations has been developed in this paper. Numerical manifold schemes integrated velocity and pressure were derived based on Galerkin-weighted residuals method as well. Mixed cover with linear polynomial function for velocity and constant function for pressure was adopted in finite element cover system. Compared with FVM and FEM, in NMM for incompressible viscous flow, the accuracy of velocity variables approximation can be improved by adopting high-order cover function, direct numerical solution of N-S equations, and continuity equation coupled velocity and pressure variables can be implemented by adopting finite cover system with mixed cover manifold element, so it can improve the solution accuracy of velocity and pressure variables.

As an application, mixed cover 4-node rectangular manifold element has been used to simulate flow around a square cylinder in a channel and past a step at low Re numbers. As to flow around a square cylinder in a channel, accurate numerical results have been presented for steady flow at $\operatorname{Re}=25,50,75,100,150$ and for unsteady flow at $\operatorname{Re}=100$, 150. The rules on streamline patterns and pressure distributions were analyzed in details. Numerical solutions were in very good agreement with the published data from very fine element meshes. Numerical tests indicate that manifold method is an effective and high-order accurate numerical method for incompressible viscous flow Navier-Stokes equations. 


\section{Acknowledgment}

Financial support for this project was provided by the National Natural Science Foundation of China (no. 50975050).

\section{References}

[1] J. H. Ferziger and M. Perić, Computational Methods for Fluid Dynamics, Springer, New York, NY, USA, 3rd edition, 1996.

[2] J. Nordström, J. Gong, E. van der Weide, and M. Svärd, "A stable and conservative high order multiblock method for the compressible Navier-Stokes equations," Journal of Computational Physics, vol. 228, no. 24, pp. 9020-9035, 2009.

[3] L. Zhang and Z. Chen, "A stabilized mixed finite element method for single-phase compressible flow," Journal of Applied Mathematics, vol. 2011, Article ID 129724, 16 pages, 2011.

[4] S. Boivin, F. Cayré, and J. M. Hérard, "A finite volume method to solve the Navier-Stokes equations for incompressible flows on unstructured meshes," International Journal of Thermal Sciences, vol. 39, no. 8, pp. 806-825, 2000.

[5] P. Poř́́zková, K. Kozel, and J. Horáček, "Numerical simulation of unsteady compressible flow in convergent channel: pressure spectral analysis," Journal of Applied Mathematics, vol. 2012, Article ID 545120, 9 pages, 2012.

[6] G. H. Shi, "Manifold method," in Proceedings of the 1st International Forum on Discontinuous Deformation Analysis E Simulation of Discontinuous Media, pp. 52-204, Berkeley, Calif, USA, 1996.

[7] G. H. Shi, "Manifold method of material analysis," in Proceedings of the 9th Army Conference on Applied Mathematics and Computing, pp. 51-76, Minneapolis, Minn, USA, 1992.

[8] K. Terada and M. Kurumatani, "Performance assessment of generalized elements in the finite cover method," Finite Elements in Analysis and Design, vol. 41, no. 2, pp. 111-132, 2004.

[9] K. Terada and M. Kurumatani, "An integrated procedure for three-dimensional structural analysis with the finite cover method," International Journal for Numerical Methods in Engineering, vol. 63, no. 15, pp. 2102-2123, 2005.

[10] K. Terada, T. Ishii, T. Kyoya, and Y. Kishino, "Finite cover method for progressive failure with cohesive zone fracture in heterogeneous solids and structures," Computational Mechanics, vol. 39, no. 2, pp. $191-$ $210,2007$.

[11] H. Y. Liu, S. R. Lv, and S. Q. Qin, "Simulation of rock shock damage evolution law with numerical manifold method," Engineering Science, vol. 19, no. 1, pp. 392-396, 2007 (Chinese).

[12] G. F. Wei and W. Feng, "Incompatible numerical manifold method based on heat exchange problem," Chinese Quarterly of Mechanics, vol. 26, no. 3, pp. 451-454, 2005 (Chinese).

[13] Z. R. Zhang, X. W. Zhang, and J. H. Yan, "Manifold method coupled velocity and pressure for NavierStokes equations and direct numerical solution of unsteady incompressible viscous flow," Computers E Fluids, vol. 39, no. 8, pp. 1353-1365, 2010.

[14] N. J. Cherry, R. Hillier, and M. E. M. P. Latour, "Unsteady measurements in a separated and reattaching flow," Journal of Fluid Mechanics, vol. 144, pp. 13-46, 1984.

[15] A. Mukhopadhyay, G. Biswas, and T. Sundararajan, "Numerical investigation of confined wakes behind a square cylinder in a channel," International Journal for Numerical Methods in Fluids, vol. 14, no. 12, pp. 1473-1484, 1992.

[16] Z. Dang, G. Xi, and S. J. Wang, "Application of the piso method in simulation of unsteady wakes behind a square cylinder," Journal of Engineering Thermophysics, vol. 20, no. 3, pp. 317-321, 1999 (Chinese).

[17] Y. Yoshida and T. Nomura, "A transient solution method for the finite element incompressible NavierStokes equations," International Journal for Numerical Methods in Fluids, vol. 5, no. 10, pp. 873-890, 1985.

[18] O. C. Zienkiewicz, S. Qu, R. L. Taylor, and S. Nakazawa, "The patch test for mixed formulations," International Journal for Numerical Methods in Engineering, vol. 23, no. 10, pp. 1873-1883, 1986. 


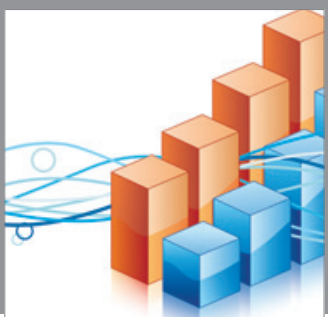

Advances in

Operations Research

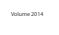

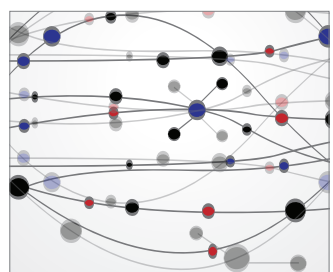

\section{The Scientific} World Journal
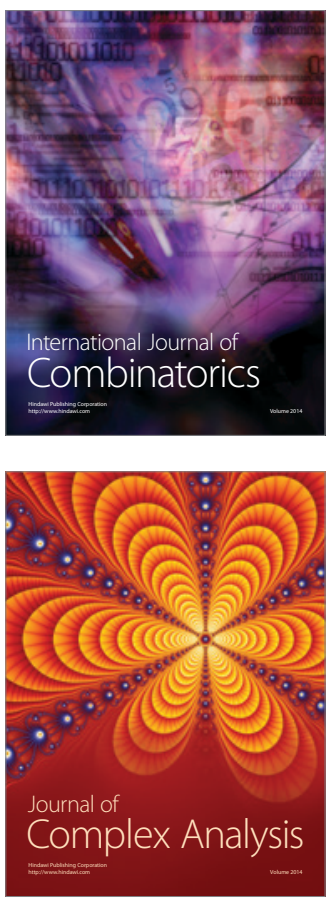

International Journal of

Mathematics and

Mathematical

Sciences
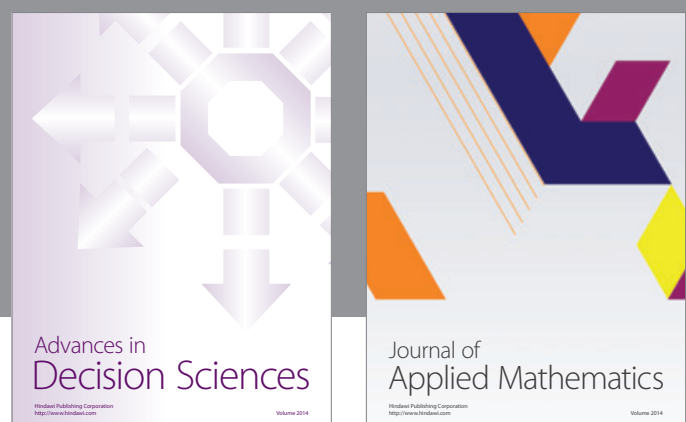

Journal of

Applied Mathematics
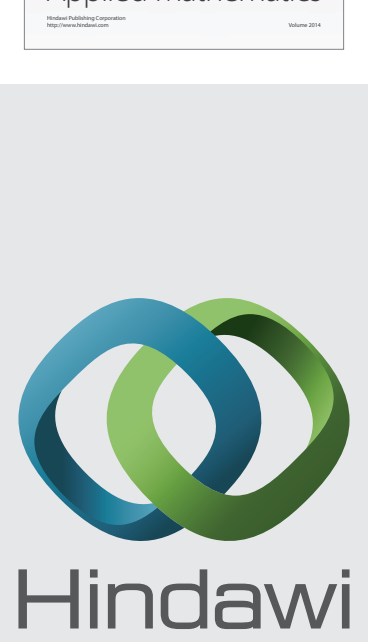

Submit your manuscripts at http://www.hindawi.com
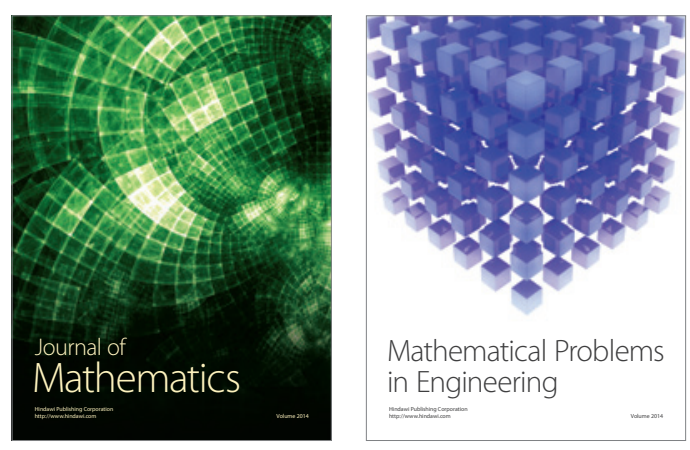

Mathematical Problems in Engineering
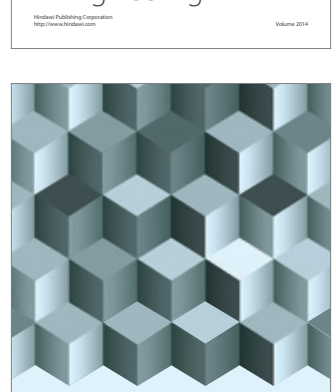

Journal of

Function Spaces
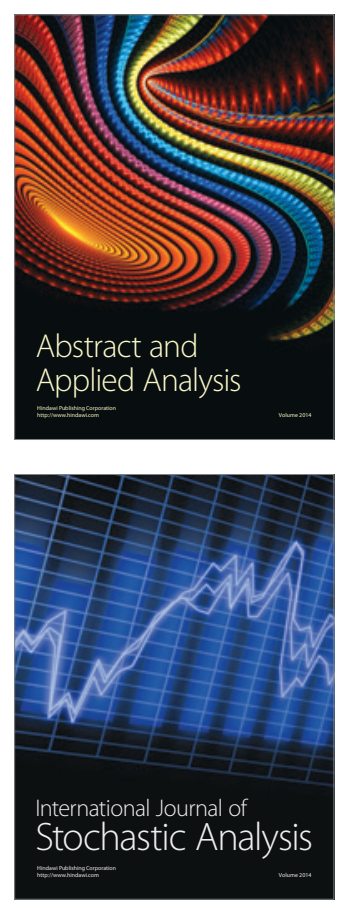

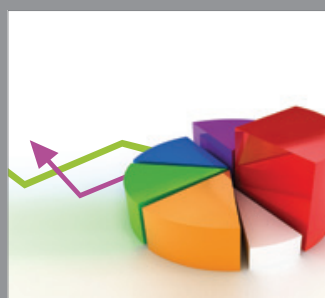

ournal of

Probability and Statistics

Promensencen
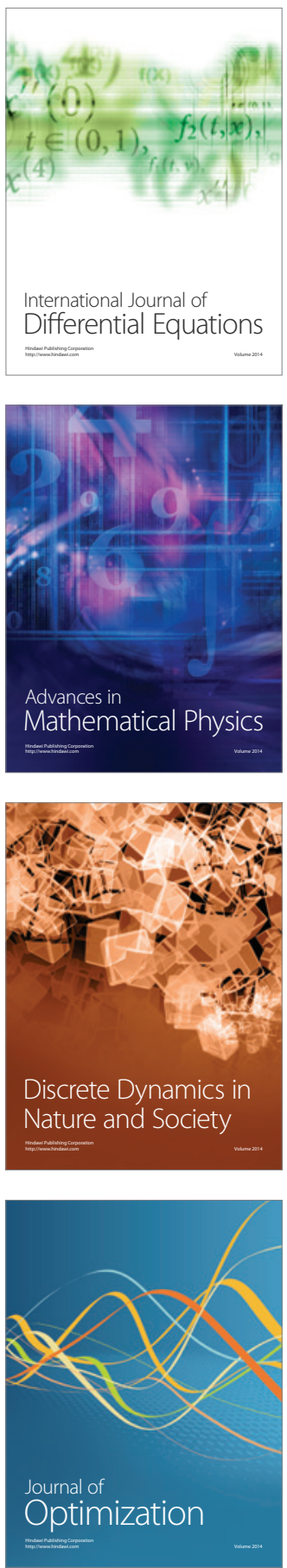